\title{
Perfil de sensibilidad de $S$. agalactiae obtenido a partir de muestras de introito vaginal y región perineal de mujeres gestantes de Medellín (Colombia)
}

\author{
Clara María Duque’, Beatriz Gómez', Diana Marcela Sánchez', \\ Olga Lucia Uribe'
}

1. Grupo de investigación Biociencias, Institución Universitaria Colegio Mayor de Antioquia

Correspondencia: clara.duque@colmayor.edu.co

Recibido:19/09/2011 - Aceptado: 21/10/2011

\begin{abstract}
Resumen
El presente trabajo tuvo como objetivo determinar el Perfil de sensibilidad de S. agalactiae obtenido a partir de muestras de introito vaginal y región perineal de mujeres gestantes de Medellín mediante un estudio descriptivo de corte en el cual se determino el perfil de sensibilidad de asilamientos de $S$. agalactiae obtenidos de muestras de introito vaginal y región perineal de mujeres gestantes de la ciudad de Medellín en el periodo 2008-20 10. Los aislamientos de S. agalactiae se recuperaron al sembrar las muestras en medio caldo ToddHewitt con suplemento de antibióticos, a partir del cual se hizo un subcultivo en agar sangre de carnero al 5\%. A los 50 aislamientos obtenidos se les evaluó el perfil de sensibilidad antimicrobiana utilizando la técnica de Kirby-Bauer, según método estandarizado por Clinical and Laboratory Standards Institute (CLSI). Se observó sensibilidad para ampicilina y vancomicina del 100\%, 25\% para tetraciclina, 72\% para eritromicina y clindamicina.
\end{abstract}

Palabras clave: S. agalactiae, Perfil de sensibilidad, mecanismo MLSB

\begin{abstract}
To determine the susceptibility profile of $S$. agalactiae isolated from vaginal cavity and perineal region from pregnant women in Medellín (Colombia)

We have carried out a descriptive study to determine the susceptibility profile of $S$. agalactiae isolates from the vaginal cavity and the perineal region from pregnant women in Medellín, between 2008 and 20 I0. The S. agalactiae isolates were obtained by first culturing samples in Todd Hewitt broth with antibiotics followed by sub-culturing in agar with Blood 5\%. The Kirby-Bauer method described by the Clinical and Laboratory Standards Institute (CLSI) was used to evaluate the antimicrobial susceptibility profile of the isolates. A susceptibility of $100 \%$ to ampicillin and vancomycin was observed, that decreased to $25 \%$ for tetracycline and $72 \%$ for erythromycin and clindamycin.
\end{abstract}

Key words: S. agalactiae, susceptibility profile, MLSB mechanism. 


\section{Introducción}

Streptococcus agalactiae es un coco Gram positivo frecuentemente asociado a infecciones en recién nacidos, con gran importancia en mujeresgestantes $\mathrm{y}$ en neonatos debido a que en mujeres en embarazadas puede dar origen a infección urinaria, corioamnionitis, endometritis, endocarditis y fiebre. Así mismo se ha identificado como el agente etiológico más frecuentemente aislado en sepsis bacteriana y meningitis neonatal(1).

La transmisión de $S$. agalactiae (SGA) de las madres colonizadas al recién nacido puede producirse en el útero o por el paso a través del canal del parto (1). En Colombia existen reportes variables de colonización materna que van desde $0 \%$ hasta un 25\% (2-6). La detección de $S$. agalactiae en mujeres embarazadas antes del parto es fundamental para prevenir la transmisión vertical y la infección del neonato.

La infección por SGA es usualmente tratada con la combinación de penicilina y gentamicina. Por consenso, las mujeres embarazadas reciben tratamiento profiláctico con penicilina o ampicilina, en el caso de presentar alergia son tratadas con eritromicina o clindamicina. De esta forma, el uso generalizado de estos antibióticos ha favorecido el incremento de la resistencia antibiótica por parte de SGA y por lo tanto una reducción en la eficacia del tratamiento tradicional. Con estos antecedentes se vuelve fundamental la vigilancia de resistencia antimicrobiana al definir los fármacos de elección y las alternativas en la profilaxis (7).

\section{Materiales y métodos}

\section{Tipo de estudio, población y muestra}

Se realizó estudio descriptivo de corte, en el cual se determino el perfil de sensibilidad de 50 asilamientos de $S$. agalactiae obtenidos de muestras de introito vaginal y región perineal de 700 mujeres gestantes de la ciudad de Medellín en el periodo 2008-2010, que consultaron en el programa de control prenatal de diferentes IPS de la ciudad. Se invitó a cada gestante a formar parte del estudio y se obtuvo consentimiento informado para participar en el mismo.
El procesamiento de la información se realizo en el programa EPI INFO.

\section{Toma de muestras clínicas, aislamiento e identificación}

A todas las participantes del estudio se le tomaron hisopados de introito vaginal y de la región anal. Las muestras fueron transportadas desde los diferentes establecimientos de salud en el medio Stuart hasta el laboratorio de investigación de la Institución Universitaria Colegio Mayor de Antioquia (IUCMA), donde las muestras fueron sembradas en el caldo ToddHewitt adicionado con ácido Nalidixico y Colistina, en atmósfera de $\mathrm{CO}_{2}$ a una temperatura de $37^{\circ} \mathrm{C}$ por 24 horas. Posteriormente, se subcultivó a agar sangre de carnero al $5 \%$ en cual se incubó en $\mathrm{CO}_{2}$ por 24 horas, las colonias compatibles con S. agalactiae, se identificaron mediante las siguientes pruebas: coloración de Gram, catalasa (reacción negativa), CAMP (reacción positiva) y aglutinación con partículas de látex, utilizando el kit comercial strepto plus B de la casa comercial Biomereux.

\section{Susceptibilidad antimicrobiana}

Se determinó mediante la técnica Kirby Bauer en agar Mueller-Hinton con 5\% de sangre de cordero con los siguientes antimicrobianos: ampicilina, clindamicina, eritromicina y tetraciclina, como inoculo se utilizó estándar $0.5 \mathrm{Mc}$ farland, las placas fueron incubadas por $24 \mathrm{~h}$ a $35^{\circ} \mathrm{C} \pm 2$ en $5 \% \mathrm{CO} 2$. Como cepa control se empleó $S$. pneumoniae ATCC 49619. Método estandarizado por Clinical and Laboratory Standards Institute (CLSI) (8).

\section{Consideraciones éticas}

El proyecto contó con la aprobación del Comité de investigaciones de la Institución Universitaria Colegio Mayor de Antioquia, teniendo en cuenta las consideraciones que garantizaron el cumplimiento de los lineamientos éticos del estudio y la obtención del consentimiento informado de los pacientes.

\section{Resultados}

Se estudiaron 700 mujeres gestantes de los estratos socioeconómicos 1, 2 y 3 de Medellín, pertene- 


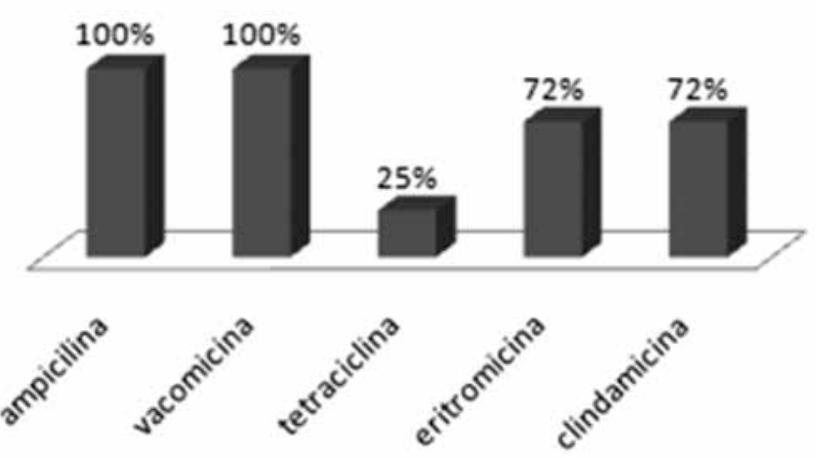

Figura 1. Perfil de sensibilidad a antibióticos de S. agalactiae.

cientes al régimen subsidiado o contributivo. En 50 $(7.14 \%)$ se aisló $S$. agalactiae.

De las 50 cepas estudiadas el 100\% fueron sensibles a ampicilina y vancomicina, $25 \%$ a tetraciclina, $72 \%$ a eritromicina y clindamicina, Figura 1.

Se determinó una resistencia a clindamicina y eritromicina del 28\%, los mecanismos observados fueron: MLSB inducible en 2 de los 14 aislamientos (14.2\%) y en $12(85.8 \%)$ MLSB constitutivo.

\section{Discusión}

Con este estudio se demuestra que la prevalencia de colonización por S.agalactiae es de $7.1 \%$ en la población estudiada, esto indica que se requiere de medidas de vigilancia epidemiológica que permitan disminuir las tasas de infección materna y de transmisión al neonato.

La prevalencia varia de acuerdo a la zona geográfica y la población objeto de estudio, como se observa en los trabajos realizados por diferentes autores donde se reportan cifras que van desde $0 \%$ hasta $25 \%$ (2-6). Igualmente en estudio realizado por Quiroga en Argentina (9) se sugiere que tanto la incidencia de la colonización como el antibiótico a emplear en la profilaxis intraparto depende del área geográfica y de las características de la población estudiada, además se afirma que la resistencia a la clindamicina y eritromicina es diversa, por lo tanto la profilaxis debería ser guiada por los perfiles de susceptibilidad antibiótica de cada región.

En este estudio se determino un 28\% de resistencia a ambos antibióticos (clindamicina y eri- tromicina). En chile en 100 cepas de $S$. agalactiae recuperadas de muestras vaginales y de región perineal de mujeres gestantes, $17(17,1 \%)$ fueron resistentes a eritromicina y 13 resistentes a clindamicina $(13,1 \%)(10)$.

Aún no existen reportes de resistencia a penicilina ni ampicilina, exceptuando el estudio realizado en el hospital Muhimbili de Tanzania en el que se reportó una resistencia de 9,4\% a penicilina y 100\% de susceptibilidad a ampicilina. (11), al igual que los otros estudios, en este trabajo se reporto sensibilidad del $100 \%$ a ambos antibióticos.

Así mismo, en un estudio realizado en Taiwanse reporto resistencia en 26 (1.3\%) de los aislamientos a levofloxacin (12), por esta razón es importante realizar seguimiento a los perfiles de sensibilidad a diferentes antibióticos en cada una de las regiones. Simoes y colaboradores en estudio realizado en Chicago reportaron $100 \%$ de sensibilidad a vancomicina, ofloxacina, ampicilina, ciprofloxacina, nitrofurantoina y penicilina; $31 \%$ fueron resistentes azitromicina y 19\% a clindamicina. (13), datos muy similares a los encontrados en el presente estudio(28\%). González, en estudio multicéntrico reporto el $12 \%$ de resistencia a eritromicina y $7 \%$ a clindamicina (14).

En un estudio realizado por Valdés el antibiograma de las cepas cultivadas mostró $100 \%$ de sensibilidad para penicilina, ampicilina y eritromicina y $70,5 \%$ para lincomicina (15), en este estudio por el contrario encontramos resistencia a eritromicina del $28 \%$.

En un estudio realizado en Argentina, de 88 aislamientos de $S$. agalactiaede muestras de orina en los que se realizó la prueba de sensibilidad a los antimicrobianos todosfueron sensibles a ampicilina, vancomicina, cloranfenicol y cefotaxima. Una cepa resultó resistente a eritromicina y otras dos mostraron sensibilidad intermedia a eritromicina y clindamicina respectivamente (16-17).

Todos estos hallazgos incluidos los del presente estudio muestran un incremento en la resistencia a macrólidos en cepas de $S$. agalactiae demostrando la necesidad de realizar un seguimiento continuo a dichos aislamientos.

El fenotipo eritromicina-clindamicina sensible fue el predominante en el estudio. Sin embargo se 
encontró una resistencia a clindamicina y eritromicina del 28\%, los mecanismos observados fueron: MLSB constitutivo en $85.8 \%$ y MLSB inducible en $14.2 \%$.

Como conclusión se determinó que el porcentaje de portadoras de $S$. agalactiae coincide con lo descrito en otros estudios. El hallazgo frecuente de cepas resistentes a eritromicina y a clindamicina indica que es recomendable la realización de pruebas de sensibilidad en los aislamientos de $S$. agalactiae en pacientes resistentes a penicilina. Así mismo, se encontró una resistencia a clindamicina y eritromicina del 28\%, los mecanismos observados fueron: MLSB constitutivo en $85.8 \%$ y MLSB inducible en $14.2 \%$

\section{Referencias}

1. RajagopalL.Understanding the regulation of Group B Streptococcal virulence factors. Future Microbiol. 2009 Mar; 4(2):201-21.

2. RJ, Baquero D. Frecuencia de aislamiento de Streptococcusagalactiaeen un grupo de embarazadas y sus productos. IATREIA. 1989;2:111-3.

3. González CP, González JE. Prevalencia de estreptococo beta hemolítico en mujeres embarazadas de alto riesgo en el Hospital Simón Bolívar de Bogotá. UCIN. 2001;2:7-15.

4. Miranda J, Sánchez I, Matar S. Detección de Streptococcusagalactiae en mujeres embarazadas del hospital San Jerónimo de Montería. InfQuincEpidemiolNac. 2002;7:

5. Restrepo A, Serna L, Vanegas C, Sarria C, Durango H, Zapata C. Prevalencia de Streptococcusagalactiae en gestantes con factores de riesgo y sus recién nacidos. Hospital Universitario San Vicente de Paúl, 2002. Infectio. 2003;7:147-52.
6. Duque CM, Gómez B, Uribe OI, et. al. Comparación de métodos para la recuperación y determinación de la prevalencia de Streptococcusagalactiae en mujeres gestantes de Medellín. Infectio. 2010; 14(2): 105-111

7. Di Bartolomeo S., Gentile M., Priore G., Valle S., Di Bella A..Streptococcus agalactiae en embarazadas. 2005, 37: 142-144.

8. Clinical and Laboratory Standars Institute. Performance Standards for Antimicrobial Susceptibility Testing 2010. M100 - S20. Vol. 30 No. 1.

9. Quiroga M, Pegels, Peryra, M. Vergara. Antibiotic susceptibility patterns and prevalence of Group B streptococcus isolated from pregnant women in Misiones, Argentina. Brazilian Journal of Microbiology (2008) 39: 245-250

10. Abarzúa F, Arias A, García P y otros. Aumento de resistencia de Streptococcusagalactiae vaginal-anal en el tercer trimestre de gestación

11. a eritromicina y clindamicina al cabo de una década de tamizaje universal. Rev Chil Infect 2011; 28 (4): 334-337

12. Joachim A, Matee M I, Massawe F A, Lyamuya E F. Maternal and neonatal colonization of group B streptococcus at Muhimbili National Hospital in Dar es Salaam, Tanzania: prevalence, risk factors and antimicrobial resistance. BMC Public Health 2009; 9: 437.

13. Wu H M, Janapatla R P, Ho Y R, Hung K H, Wu C W, Yan J J, et al. Emergence of fluoroquinolone resistance in group $\mathrm{B}$ streptococcal isolates in Taiwan. Antimicrob Agents Chemother 2008; 52: 1888-90.

14. Simoes JA, Aroutcheva AA, Heimler I, Faro S. Antibiotic resistance patterns of group B streptococcal clinical isolates. Infect Dis ObstetGynecol 2004; 12: 1-8.

15. González JJ, Andreu A. Susceptibility of vertically transmitted Group B streptococci to antimicrobial agents. Multicenterstudy. EnfermInfeccMicrobiolClin. 2004 May; 22(5):286-91.

16. Valdes r, Enrique et al. Prevalencia de colonización por Streptococcus agalactiae (grupo B) durante el embarazo pesquisado en medio de cultivo selectivo. Rev. chil. obstet. ginecol. 2004, vol.69, n.2

17. ViegasCaetano J y col. Detección y caracterización de Streptococcusagalactiae en muestras para urocultivo. ActaBioquímClínLatinoam 2004; 38 (4): 459-63

18. Metsvaht T, Oselin K, Ilmoja ML, Anier K, Lutsar I .Pharmacokinetics of penicillin $\mathrm{g}$ in very-low-birth-weight neonates. Antimicrob Agents Chemother. 2007 Jun; 51(6):1995-2000.
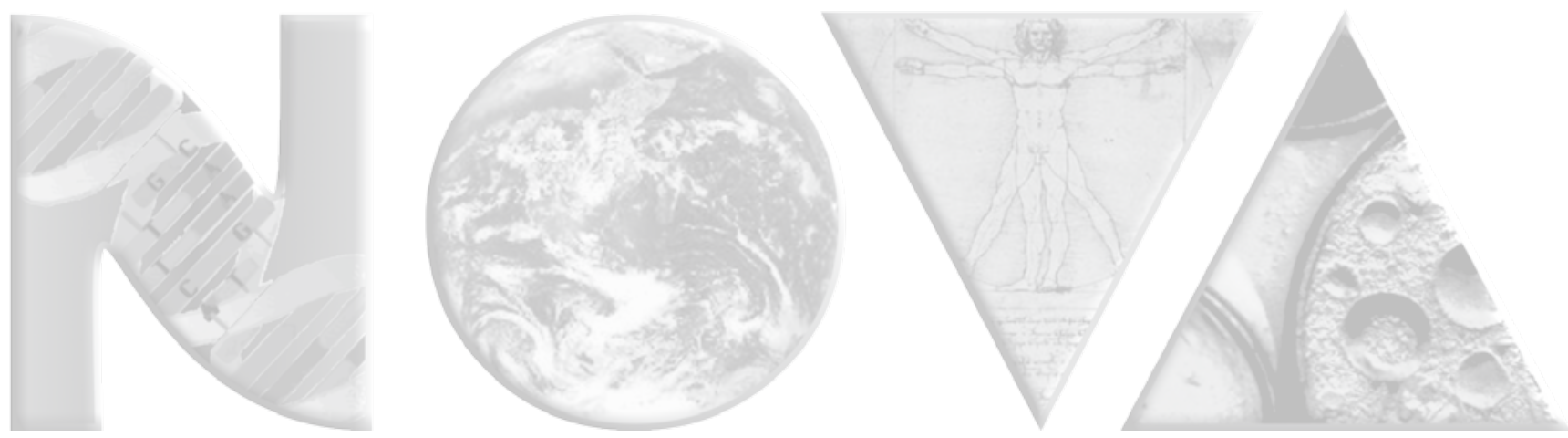